\title{
Spectral analysis of a wave equation with Kelvin-Voigt damping
}

\author{
Bao-Zhu Guo ${ }^{1,2, *}$, Jun-Min Wang ${ }^{3, * *}$, and Guo-Dong Zhang ${ }^{4}$ \\ ${ }^{1}$ Academy of Mathematics and Systems Science, Academia Sinica, Beijing 100190, P.R. China \\ 2 School of Mathematical Sciences, Shanxi University, Taiyuan 030006, P.R. China \\ ${ }^{3}$ Department of Mathematics, Beijing Institute of Technology, Beijing 100081, P.R. China \\ ${ }^{4}$ School of Computational and Applied Mathematics, University of the Witwatersrand, Wits 2050, Johannesburg, \\ South Africa
}

Received 23 March 2009, revised 13 October 2009, accepted 12 January 2010

Published online 24 March 2010

Key words Wave equation, spectrum, Kelvin-Voigt damping.

A vibrating system with some kind of internal damping represents a distributed or passive control. In this article, a wave equation with clamped boundary conditions and internal Kelvin-Voigt damping is considered. It is shown that the spectrum of the system operator is composed of two parts: point spectrum and continuous spectrum. The point spectrum consists of isolated eigenvalues of finite algebraic multiplicity, and the continuous spectrum that is identical to the essential spectrum is an interval on the left real axis. The asymptotic behavior of eigenvalues is presented.

(c) 2010 WILEY-VCH Verlag GmbH \& Co. KGaA, Weinheim

\section{Introduction}

Owing to huge applications of smart materials in modern technology, there has been an abundance of literature on the study of elastic systems with viscoelastic damping ( [1]). When a smart material is patched in an elastic structure, the Young's modulus, the mass density and the damping coefficients are changed accordingly. Practically, two types of viscoelastic damping are usually used. One is the Botzmann damping and another is the Kelvin-Voigt damping. These kinds of damping, on the one hand, make the distributed control practically realizable, and on the other hand, bring some new mathematical challenges that attract increasing research interests. For the controllability of this kind of systems, we refer to [14]. The stability can be found in $[4,15-17]$. Very recently, an interesting Riesz basis property was developed for such a system in [13]. However, for these works aforementioned, only partial solutions to the distribution of the vibration frequencies were presented. This is an unfortunate situation since it has been shown for many other elastic systems in $[9,10,18,21]$ that for a vibrating system, the vibration frequencies could determine all dynamic behaviors of the system. The reason for this situation occurs is that for a viscoelastic system, the resolvent of system operator is not compact anymore, which is sharp contrast with that discussed in $[9,10,18,21]$. Nevertheless, a viscoelastic system with constant coefficients still shows the valid of Riesz basis property due to the fact that the continuous spectrum is the limit set of point spectrum [11]. But for the viscoelastic systems with variable coefficients, situations are quite different even in one-dimensional cases. In [20], the asymptotic behavior for a one-dimensional wave equation with local Kelvin-Voigt damping was developed. In [22], the essential spectrum of a system operator arising in viscoelastic system with local Kelvin-Voigt damping was analyzed. In [12], the spectrum of a general second order system was discussed. In this paper, we study a one-dimensional wave equation with clamped boundary condition and internal Kelvin-Voigt damping. It is shown that the spectrum of this system operator is composed of two parts: point spectrum and continuous spectrum. The point spectrum consists of isolated eigenvalues of finite algebraic multiplicity, and the continuous spectrum that is identical to the essential spectrum is an interval on the left real axis under the analyticity assumption of coefficients. The asymptotic behavior of eigenvalues is also presented.

This paper is organized as follows. In next section, Sect. 2, we formulate the problem into an abstract evolution equation in the state space. Sect. 3 is devoted to the analysis of continuous spectrum. The asymptotic expression for eigenvalues is presented in Sect. 4.

* Corresponding author E-mail: bzguo@iss.ac.cn

** E-mail: wangjc@graduate.hku.hk 


\section{System operator setup}

Consider a one-dimensional wave equation with Kelvin-Voigt damping and clamped boundary conditions:

$$
\left\{\begin{array}{l}
\rho(x) y_{t t}(x, t)-\left(a(x) y_{x}(x, t)+b(x) y_{x t}(x, t)\right)^{\prime}=0,0<x<1, t>0, \\
y(0, t)=y(1, t)=0 \\
y(x, 0)=y_{0}(x), y_{t}(x, 0)=y_{1}(x)
\end{array}\right.
$$

where the continuous function $b(\cdot) \geq 0$ is the damping function, and the continuous functions $\rho(\cdot), a(\cdot)>0$ are system parameter functions in spacial variable. Hereafter, we use prime " $"$ " to represent the derivative with respect to $x$. The system energy is given by

$$
E(t)=\frac{1}{2} \int_{0}^{1}\left[a(x)\left|y_{x}(x, t)\right|^{2}+\rho(x)\left|y_{t}(x, t)\right|^{2}\right] d x .
$$

For any positive continuous function $\rho$, set $L_{\rho}^{2}=L^{2}(0,1)$ with norm $\|f\|_{L_{\rho}^{2}}^{2}=\int_{0}^{1} \rho(x)|f(x)|^{2} d x$ and $V=H_{0}^{1}(0,1)$, the first order Sobolev space with zero boundary values. We consider the system (2.1) in the energy state Hilbert space $\mathcal{H}=V \times L_{\rho}^{2}$ with the inner product:

$$
\left\langle\left(f_{1}, g_{1}\right),\left(f_{2}, g_{2}\right)\right\rangle=\int_{0}^{1}\left[a(x) f_{1}^{\prime}(x) \overline{f_{2}^{\prime}(x)}+\rho(x) g_{1}(x) \overline{g_{2}(x)}\right] d x, \quad \forall\left(f_{i}, g_{i}\right) \in \mathcal{H}, i=1,2 .
$$

Define the system operator $\mathcal{A}: D(\mathcal{A})(\subset \mathcal{H}) \rightarrow \mathcal{H}$ as

$$
\left\{\begin{array}{l}
\mathcal{A}(f, g)=\left(g, \frac{1}{\rho}\left(a f^{\prime}+b g^{\prime}\right)^{\prime}\right), \\
D(\mathcal{A})=\left\{(f, g) \in H_{0}^{1}(0,1) \times H_{0}^{1}(0,1) \mid a f^{\prime}+b g^{\prime} \in H^{1}(0,1)\right\} .
\end{array}\right.
$$

Then (2.1) can be formulated into an abstract evolution equation in $\mathcal{H}$ :

$$
\frac{\mathrm{d}}{\mathrm{d} t} Y(t)=\mathcal{A} Y(t), \quad Y(0)=Y_{0},
$$

where $Y(t)=\left(y(\cdot, t), y_{t}(\cdot, t)\right)$ is the state variable and $Y_{0}=\left(y_{0}(\cdot), y_{1}(\cdot)\right)$ is the initial value.

The following Lemma 2.1 is straightforward.

Lemma 2.1. Let $\mathcal{A}$ be defined by (2.4). Then its adjoint $\mathcal{A}^{*}$ has the following form:

$$
\left\{\begin{array}{l}
\mathcal{A}^{*}(f, g)=\left(-g,-\frac{1}{\rho}\left(a f^{\prime}-b g^{\prime}\right)^{\prime}\right) \\
D\left(\mathcal{A}^{*}\right)=\left\{(f, g) \in V \times V \mid a f^{\prime}-b g^{\prime} \in H^{1}(0,1)\right\} .
\end{array}\right.
$$

Proposition 2.2. Let $\mathcal{A}$ and $\mathcal{A}^{*}$ be given by (2.4) and (2.6), respectively. Then $\mathcal{A}$ and $\mathcal{A}^{*}$ are dissipative, and hence $\mathcal{A}$ generates a $C_{0}$-semigroup of contractions on $\mathcal{H}$.

Proof. For any $(f, g) \in D(\mathcal{A})$, we have

$$
\begin{aligned}
\langle\mathcal{A}(f, g),(f, g)\rangle & =\left\langle\left(g, \frac{1}{\rho}\left(a f^{\prime}+b g^{\prime}\right)^{\prime}\right),(f, g)\right\rangle \\
& =\int_{0}^{1}\left[a(x) g^{\prime}(x) \overline{f^{\prime}(x)}+\left(a(x) f^{\prime}(x)+b(x) g^{\prime}(x)\right)^{\prime} \overline{g(x)}\right] d x \\
& =\int_{0}^{1}\left[a(x) g^{\prime}(x) \overline{f^{\prime}(x)}-a(x) f^{\prime}(x) \overline{g^{\prime}(x)}\right] d x-\int_{0}^{1} b(x)\left|g^{\prime}(x)\right|^{2} d x,
\end{aligned}
$$

and hence

$$
\operatorname{Re}\langle\mathcal{A}(f, g),(f, g)\rangle=-\int_{0}^{1} b(x)\left|g^{\prime}(x)\right|^{2} d x \leq 0 .
$$


Similarly for any $(u, v) \in D\left(\mathcal{A}^{*}\right)$,

$$
\begin{aligned}
\left\langle\mathcal{A}^{*}(u, v),(u, v)\right\rangle & =\left\langle\left(-v,-\frac{1}{\rho}\left(a u^{\prime}-b v^{\prime}\right)^{\prime}\right),(u, v)\right\rangle \\
& =\int_{0}^{1}\left[-a(x) v^{\prime}(x) \overline{u^{\prime}(x)}-\left(a(x) u^{\prime}(x)-b(x) v^{\prime}(x)\right)^{\prime} \overline{v(x)}\right] d x \\
& =\int_{0}^{1}\left[-a(x) v^{\prime}(x) \overline{u^{\prime}(x)}+a(x) u^{\prime}(x) \overline{v^{\prime}(x)}\right] d x-\int_{0}^{1} b(x)\left|v^{\prime}(x)\right|^{2} d x,
\end{aligned}
$$

and hence

$$
\operatorname{Re}\left\langle\mathcal{A}^{*}(u, v),(u, v)\right\rangle=-\int_{0}^{1} b(x)\left|v^{\prime}(x)\right|^{2} d x \leq 0 .
$$

Therefore, both $\mathcal{A}$ and $\mathcal{A}^{*}$ are dissipative. By the Lumer-Phillips Theorem, $\mathcal{A}$ generates a $C_{0}$-semigroup of contractions on $\mathcal{H}$.

\section{Continuous spectrum of the system operator}

In this section, we consider the spectrum of $\mathcal{A}$. First, let us formulate the eigenvalue problem. Suppose $\mathcal{A}(f, g)=\lambda(f, g)$ with $(f, g) \in D(\mathcal{A})$ and $(f, g) \neq 0$. Then $g=\lambda f$ and $f \in H_{0}^{1}(0,1)$ satisfies

$$
\left\{\begin{array}{l}
\left((a(x)+\lambda b(x)) f^{\prime}(x)\right)^{\prime}=\lambda^{2} \rho(x) f(x) \\
f(0)=f(1)=0
\end{array}\right.
$$

The Theorem 3.1 following shows that the set $\sigma_{r}(\mathcal{A})$ of residual spectrum of $\mathcal{A}$ is empty.

Theorem 3.1. $\sigma_{r}(\mathcal{A})=\emptyset$.

Proof. Since $\lambda \in \sigma_{r}(\mathcal{A})$ if and only if $\bar{\lambda} \in \sigma_{p}\left(\mathcal{A}^{*}\right)$, it suffices to show that $\sigma_{p}(\mathcal{A})=\sigma_{p}\left(\mathcal{A}^{*}\right)$. Suppose $\mathcal{A}^{*}(f, g)=$ $\lambda(f, g)$ for some $(f, g) \in D\left(\mathcal{A}^{*}\right)$ and $(f, g) \neq 0$. Then $g=-\lambda f$ and $f$ satisfies

$$
\left\{\begin{array}{l}
\left(a(x) f^{\prime}(x)+\lambda b(x) f^{\prime}(x)\right)^{\prime}=\lambda^{2} \rho(x) f(x), \\
f(0)=f(1)=0 .
\end{array}\right.
$$

It is seen that (3.2) is the same with (3.1). Hence, $\lambda \in \sigma_{p}\left(\mathcal{A}^{*}\right)$ if and only if $\lambda \in \sigma_{p}(\mathcal{A})$. Since the eigenvalues of $\mathcal{A}^{*}$ are symmetric with real axis, we have $\sigma_{r}(\mathcal{A})=\emptyset$.

Proposition 3.2. Let $\mathcal{A}$ be defined by (2.4). Then $0 \in \rho(\mathcal{A})$ and $\mathcal{A}^{-1}$ is given by

$$
\mathcal{A}^{-1}\left(\begin{array}{c}
f \\
g
\end{array}\right)(x)=\left(\begin{array}{c}
g_{1}(x)-\int_{0}^{x} \frac{b(\tau)}{a(\tau)} f^{\prime}(\tau) d \tau+\frac{a_{1}(x)}{a_{1}(1)}\left[\int_{0}^{1} \frac{b(\tau)}{a(\tau)} f^{\prime}(\tau) d \tau-g_{1}(1)\right] \\
f(x)
\end{array}\right),
$$

where

$$
g_{1}(x)=\int_{0}^{x} \frac{1}{a(\tau)}\left[\int_{0}^{\tau} \rho(s) g(s) d s\right] d \tau, \quad a_{1}(x)=\int_{0}^{x} \frac{1}{a(\tau)} d \tau .
$$

Proof. $\quad$ Let $(f, g) \in \mathcal{H}$. By $\mathcal{A}(\phi, \psi)=(f, g)$, we have

$$
\psi=f, \frac{1}{\rho}\left(a \phi^{\prime}+b \psi^{\prime}\right)^{\prime}=g .
$$

These together with the boundary conditions show that

$$
\left\{\begin{array}{l}
\left(a(x) \phi^{\prime}(x)+b(x) f^{\prime}(x)\right)^{\prime}=\rho(x) g(x), \\
\phi(0)=\phi(1)=0 .
\end{array}\right.
$$


A direct computation gives

$$
\phi(x)=g_{1}(x)-\int_{0}^{x} \frac{b(\tau)}{a(\tau)} f^{\prime}(\tau) d \tau+C_{1} a_{1}(x),
$$

where $g_{1}(x), a_{1}(x)$ are given by (3.4), and $C_{1}$ satisfies

$$
C_{1}=\left[a \phi^{\prime}+b f^{\prime}\right](0) \text {. }
$$

Using the boundary condition $\phi(1)=0$, it gives

$$
C_{1}=\frac{1}{a_{1}(1)}\left[\int_{0}^{1} \frac{b(\tau)}{a(\tau)} f^{\prime}(\tau) d \tau-g_{1}(1)\right] .
$$

Therefore

$$
\phi(x)=g_{1}(x)-\int_{0}^{x} \frac{b(\tau)}{a(\tau)} f^{\prime}(\tau) d \tau+\frac{a_{1}(x)}{a_{1}(1)}\left[\int_{0}^{1} \frac{b(\tau)}{a(\tau)} f^{\prime}(\tau) d \tau-g_{1}(1)\right] .
$$

This together with (3.5) gives (3.3). The proof is complete.

The following Definition 3.3 comes from [8, p. 373].

Definition 3.3. Let $T$ be a closed linear operator in a Hilbert space. The set of complex numbers $\lambda$ is called the essential spectrum of $T$, and is denoted by $\sigma_{\text {ess }}(T)$, if one of the following three conditions is satisfied:

(i). $\mathcal{R}(\lambda I-T)$, the range of $\lambda I-T$, is not closed.

(ii). $\operatorname{dim} \mathcal{N}(\lambda I-T)=\infty$, here $\mathcal{N}(\lambda I-T)$ denotes the null space of $\lambda I-T$.

(iii). $\operatorname{dim}(\mathcal{R}(\lambda I-T))^{\perp}=\infty$, here $(\mathcal{R}(\lambda I-T))^{\perp}$ is the orthogonal complement space of range $\mathcal{R}(\lambda I-T)$ of $\lambda I-T$.

Notice that if $T$ is densely defined, then (iii) of Definition 3.3 can be replaced by $\operatorname{dim} \mathcal{N}\left(\bar{\lambda} I-T^{*}\right)=\infty$.

The following Proposition 3.4 is a direct consequence of Corollary 4.4 of ( [8, p.378].

Proposition 3.4. Let $T$ be a closed linear operator in a Hilbert space and G a compact operator. Then

$$
\sigma_{e s s}(T)=\sigma_{e s s}(T+G) \text {. }
$$

Now we go back to our problem. Define a bounded linear operator $\mathcal{D}: V \rightarrow L_{a}^{2}$ by

$$
(\mathcal{D} f)(x)=f^{\prime}(x), \forall f \in V=H_{0}^{1}(0,1) .
$$

The following Lemma 3.5 is straightforward.

Lemma 3.5. Let $\mathcal{D}$ be defined by (3.6). Then the following assertions hold:

(i) For any $\phi \in V,\|\mathcal{D} \phi\|_{L_{a}^{2}}=\|\phi\|_{V}$.

(ii) The range of $\mathcal{D}$,

$$
\mathcal{R}(\mathcal{D})=\left\{f \in L_{a}^{2} \mid\left\langle f(\cdot), \frac{1}{a(\cdot)}\right\rangle_{L_{a}^{2}}=0\right\}
$$

is a closed subspace of $L_{a}^{2}$.

(iii) $\mathcal{D}^{-1}$ is a bounded linear operator from $\mathcal{R}(\mathcal{D})$ onto $V$ given by

$$
\mathcal{D}^{-1} f(x)=\int_{0}^{x} f(\tau) d \tau, \quad \forall f \in \mathcal{R}(\mathcal{D}) .
$$

Let $\mathcal{H}_{1}=\mathcal{R}(\mathcal{D}) \times L_{\rho}^{2}$ with the same inner product defined by (2.3). Define the linear operator $\mathcal{T}: \mathcal{H} \rightarrow \mathcal{H}_{1}$ by

$$
\mathcal{T}(\phi, \psi)=(\mathcal{D} \phi, \psi)=\left(\phi^{\prime}, \psi\right), \forall(\phi, \psi) \in \mathcal{H}
$$

Then, it is easy to see that

$$
\mathcal{T}^{-1}(f, g)=\left(\mathcal{D}^{-1} f, g\right), \quad \forall(f, g) \in \mathcal{H}_{1}
$$


and

$$
\|\mathcal{T}(\phi, \psi)\|_{\mathcal{H}_{1}}^{2}=\int_{0}^{1}\left[a(x)\left|\phi^{\prime}(x)\right|^{2}+\rho(x)|\psi(x)|^{2}\right] d x=\|(\phi, \psi)\|_{\mathcal{H}}^{2}, \quad \forall(\phi, \psi) \in \mathcal{H} .
$$

Define a linear operator $\widetilde{\mathcal{A}}: D(\widetilde{\mathcal{A}})\left(\subset \mathcal{H}_{1}\right) \rightarrow \mathcal{H}_{1}$ by

$$
\widetilde{\mathcal{A}}=\mathcal{T} \mathcal{A} \mathcal{T}^{-1}
$$

Then $\tilde{\mathcal{A}}$ is explicitly given by

$$
\left\{\begin{array}{l}
\widetilde{\mathcal{A}}(\phi, \psi)=\left(\psi^{\prime}, \frac{1}{\rho}\left(a \phi+b \psi^{\prime}\right)^{\prime}\right), \\
D(\widetilde{\mathcal{A}})=\left\{(\phi, \psi) \in \mathcal{R}(\mathcal{D}) \times H_{0}^{1}(0,1) \mid a \phi+b \psi^{\prime} \in H^{1}(0,1)\right\} .
\end{array}\right.
$$

By (3.10), we have Lemma 3.6 following.

Lemma 3.6. $\sigma(\mathcal{A})=\sigma(\widetilde{\mathcal{A}})$.

Proposition 3.7. Let $\widetilde{\mathcal{A}}$ be defined by (3.11). Then $\widetilde{\mathcal{A}}^{-1}$ exists and has the following expression:

$$
\widetilde{\mathcal{A}}^{-1}\left(\begin{array}{l}
f \\
g
\end{array}\right)=\mathcal{P}\left(\begin{array}{l}
f \\
g
\end{array}\right)+\mathcal{Q}\left(\begin{array}{l}
f \\
g
\end{array}\right), \quad \forall(f, g) \in \mathcal{H}_{1}
$$

where $\mathcal{P}$ and $\mathcal{Q}$ are bounded operators on $\mathcal{H}_{1}$ and have the following expressions, respectively: for each $(f, g) \in \mathcal{H}_{1}$,

$$
\mathcal{P}\left(\begin{array}{c}
f \\
g
\end{array}\right)(x)=\left(\begin{array}{c}
\frac{1}{a(x)} \int_{0}^{x} \rho(s) g(s) d s-\frac{g_{1}(1)}{a_{1}(1)} \frac{1}{a(x)} \\
\int_{0}^{x} f(\tau) d \tau
\end{array}\right)
$$

and

$$
\mathcal{Q}\left(\begin{array}{c}
f \\
g
\end{array}\right)(x)=\left(\begin{array}{c}
-\frac{b(x)}{a(x)} f(x)+\frac{1}{a_{1}(1)} \frac{1}{a(x)} \int_{0}^{1} \frac{b(\tau)}{a(\tau)} f(\tau) d \tau \\
0
\end{array}\right)
$$

Moreover,

(i) $\mathcal{P}$ is compact and skew-adjoint on $\mathcal{H}_{1}$;

(ii) $\mathcal{Q}$ is self-adjoint on $\mathcal{H}_{1}$, and its essential spectrum is given by

$$
\sigma_{\text {ess }}(\mathcal{Q})=\{0\} \cup\{\lambda \in \mathbb{C} \mid \lambda a(\xi)+b(\xi)=0 \text { for some } \xi \in[0,1]\}
$$

Proof. $\quad$ Since $\widetilde{\mathcal{A}}=\mathcal{T} \mathcal{A} \mathcal{T}^{-1}, \widetilde{\mathcal{A}}^{-1}$ exists and $\widetilde{\mathcal{A}}^{-1}=\mathcal{T} \mathcal{A}^{-1} \mathcal{T}^{-1}$. For any $(f, g) \in \mathcal{H}_{1}$,

$$
\begin{aligned}
\widetilde{\mathcal{A}}^{-1}\left(\begin{array}{l}
f \\
g
\end{array}\right)(x) & =\mathcal{T} \mathcal{A}^{-1} \mathcal{T}^{-1}\left(\begin{array}{l}
f \\
g
\end{array}\right)(x)=\mathcal{T} \mathcal{A}^{-1}\left(\begin{array}{c}
\int_{0}^{x} f(\tau) d \tau \\
g(x)
\end{array}\right) \\
& =\mathcal{T}\left(\begin{array}{c}
g_{1}(x)-\int_{0}^{x} \frac{b(\tau)}{a(\tau)} f(\tau) d \tau+\frac{a_{1}(x)}{a_{1}(1)}\left[\int_{0}^{1} \frac{b(\tau)}{a(\tau)} f(\tau) d \tau-g_{1}(1)\right] \\
\int_{0}^{x} f(\tau) d \tau
\end{array}\right) \\
& =\left(\begin{array}{c}
\frac{1}{a(x)} \int_{0}^{x} \rho(s) g(s) d s-\frac{b(x)}{a(x)} f(x)+\frac{1}{a_{1}(1)} \frac{1}{a(x)}\left[\int_{0}^{1} \frac{b(\tau)}{a(\tau)} f(\tau) d \tau-g_{1}(1)\right] \\
\int_{0}^{x} f(\tau) d \tau
\end{array}\right)
\end{aligned}
$$




$$
=\mathcal{P}\left(\begin{array}{l}
f \\
g
\end{array}\right)(x)+\mathcal{Q}\left(\begin{array}{l}
f \\
g
\end{array}\right)(x),
$$

where $\mathcal{P}$ and $\mathcal{Q}$ are defined by (3.13) and (3.14), respectively.

Notice that when $b(\cdot) \equiv 0, \mathcal{A}$ is skew-adjoint and is of compact resolvent. So is for $\widetilde{\mathcal{A}}$ when $b(\cdot) \equiv 0$, and in this case, $\widetilde{\mathcal{A}}^{-1}=\mathcal{P}$. Hence, $\mathcal{P}$ is compact and skew-adjoint on $\mathcal{H}_{1}$. (i) is thus proved.

Next we prove (ii). We first prove that $\mathcal{Q}$ is self-adjoint. Actually, for any $(f, g),(u, v) \in \mathcal{H}_{1}$, by $(3.7), f, u \in \mathcal{R}(\mathcal{D})$,

$$
\int_{0}^{1} f(x) d x=\int_{0}^{1} u(x) d x=0
$$

and hence

$$
\begin{aligned}
\langle\mathcal{Q}(f, g),(u, v)\rangle_{\mathcal{H}_{1}} & =\int_{0}^{1} a(x)\left[-\frac{b(x)}{a(x)} f(x)+\frac{1}{a_{1}(1)} \frac{1}{a(x)} \int_{0}^{1} \frac{b(\tau)}{a(\tau)} f(\tau) d \tau\right] \overline{u(x)} d x \\
& =-\int_{0}^{1} b(x) f(x) \overline{u(x)} d x \\
& =\langle(f, g), \mathcal{Q}(u, v)\rangle_{\mathcal{H}_{1}},
\end{aligned}
$$

which shows that $\mathcal{Q}$ is self-adjoint on $\mathcal{H}_{1}$.

Now we show

$$
\sigma(\mathcal{Q})=\{0\} \cup\{\lambda \in \mathbb{C} \mid \lambda a(\xi)+b(\xi)=0 \text { for some } \xi \in[0,1]\} .
$$

Let $\lambda \in \mathbb{C}$. For any $(u, v) \in \mathcal{H}_{1}$, consider the equation

$$
(\lambda I-\mathcal{Q})(f, g)=(u, v),
$$

which is equivalent to $\lambda g(x)=v(x)$ and $f$ satisfies

$$
\lambda f(x)+\frac{b(x)}{a(x)} f(x)-\frac{1}{a_{1}(1)} \frac{1}{a(x)} \int_{0}^{1} \frac{b(\tau)}{a(\tau)} f(\tau) d \tau=u(x) .
$$

Since $\left\langle u(\cdot), \frac{1}{a(\cdot)}\right\rangle_{L_{a}^{2}}=0$, if (3.17) admits a solution, integrating both sides of (3.17) over [0,1] shows that it must have $\left\langle\lambda f(\cdot), \frac{1}{a(\cdot)}\right\rangle_{L_{a}^{2}}=0$.

When $\lambda \neq 0$ and $\lambda+\frac{b(x)}{a(x)} \neq 0$ for any $x \in[0,1], g(x)=\lambda^{-1} v(x)$ and it follows from (3.17) that

$$
f(x)=\frac{a(x)}{\lambda a(x)+b(x)}\left[u(x)+\frac{1}{a_{1}(1)} \frac{1}{a(x)} \int_{0}^{1} \frac{b(\tau)}{a(\tau)} f(\tau) d \tau\right] .
$$

A direct computation gives

$$
\int_{0}^{1} \frac{b(\tau)}{a(\tau)} f(\tau) d \tau=\frac{1}{\lambda} a_{1}(1)\left(\int_{0}^{1} \frac{1}{\lambda a(\tau)+b(\tau)} d \tau\right)^{-1} \int_{0}^{1} \frac{b(\tau) u(\tau)}{\lambda a(\tau)+b(\tau)} d \tau
$$

Hence,

$$
f(x)=\frac{1}{\lambda a(x)+b(x)}\left[a(x) u(x)+\frac{1}{\lambda} \int_{0}^{1} \frac{b(\tau) u(\tau)}{\lambda a(\tau)+b(\tau)} d \tau\left(\int_{0}^{1} \frac{1}{\lambda a(\tau)+b(\tau)} d \tau\right)^{-1}\right] .
$$

So $(f, g) \in \mathcal{H}_{1}$. Therefore $\lambda \in \rho(\mathcal{Q})$, which implies that

$$
\sigma(\mathcal{Q})=\mathbb{C} \backslash \rho(\mathcal{Q}) \subseteq\{0\} \cup\{\lambda \in \mathbb{C} \mid \lambda a(\xi)+b(\xi)=0 \text { for some } \xi \in[0,1]\} .
$$


Moreover, when $\lambda=0$, since for each $(f, g) \in \mathcal{H}_{1}$,

$$
\left[(\lambda I-\mathcal{Q})\left(\begin{array}{c}
f \\
g
\end{array}\right)\right](x)=\left(\begin{array}{c}
\frac{\lambda a(x)+b(x)}{a(x)} f(x)-\frac{1}{a_{1}(1)} \frac{1}{a(x)} \int_{0}^{1} \frac{b(\tau)}{a(\tau)} f(\tau) d \tau \\
\lambda g(x)
\end{array}\right)
$$

it has $\{0\} \times L_{\rho}^{2} \subset \mathcal{N}(\mathcal{Q})$. Hence $\operatorname{dim} \mathcal{N}(\mathcal{Q})=\infty$ and by Definition 3.3,

$$
0 \in \sigma_{e s s}(\mathcal{Q}) \text {. }
$$

If $\lambda \neq 0$ and $\lambda a(\xi)+b(\xi)=0$ for some $\xi \in[0,1]$, we claim that $\mathcal{R}(\lambda I-\mathcal{Q}) \neq \mathcal{H}_{1}$. In fact, define

$$
E_{\lambda}=\{x \in[0,1] \mid \lambda a(x)+b(x)=0\} .
$$

If the measure of $E_{\lambda}$ is nonzero and (3.17) admits a solution, it must have

$$
u(x)=C / a(x) \text { in } E_{\lambda} \text { for some constant } C .
$$

Obviously, such a function cannot represent all functions of $\mathcal{R}(\mathcal{D})$ on $E_{\lambda}$, that is $\mathcal{R}(\lambda I-\mathcal{Q}) \neq \mathcal{H}_{1}$. Now suppose that the measure of $E_{\lambda}$ is zero and (3.17) has solution $f \in \mathcal{R}(\mathcal{D})$ for any $u \in \mathcal{R}(\mathcal{D})$. Then $f$ must be of the form (3.18). Take special $u \in \mathcal{R}(\mathcal{D})$ in (3.18) as following

$$
u(x)= \begin{cases}\frac{1}{\sqrt[3]{x-\xi}}, & x \in E_{1} \\ \frac{-1}{1-\operatorname{mes}\left(E_{1}\right)} \int_{E_{1}} \frac{1}{\sqrt[3]{x-\xi}} d x, & x \in[0,1] \backslash E_{1},\end{cases}
$$

where $E_{1} \subset[0,1]$ is a given small closed interval containing $\xi$, and $\operatorname{mes}\left(E_{1}\right)$ is the measure of $E_{1}, 0<\operatorname{mes}\left(E_{1}\right)<1$. Obviously, for this special $u$, there exists a closed interval $E_{2} \subset E_{1}, \xi \in E_{2}$, such that the corresponding solution $f$ satisfies

$$
\left|a(x) u(x)+\frac{1}{a_{1}(1)} \int_{0}^{1} \frac{b(\tau)}{a(\tau)} f(\tau) d \tau\right|>1, x \in E_{2},
$$

and hence by (3.18),

$$
\left\|\frac{1}{\lambda a+b}\right\|_{L^{2}\left(E_{2}\right)} \leq\|f\|_{L^{2}\left(E_{2}\right)}<\infty
$$

which means that $\frac{1}{\lambda a+b} \in L^{2}\left(E_{2}\right)$. This fact together with (3.18) shows that

$$
\frac{a \widetilde{u}}{\lambda a+b} \in L^{2}\left(E_{2}\right), \forall \widetilde{u} \in L^{2}\left(E_{2}\right)
$$

Define the multiplication operator $\mathcal{F}: L^{2}\left(E_{2}\right) \rightarrow L^{2}\left(E_{2}\right)$ by

$$
(\mathcal{F} \widetilde{u})(x)=\frac{a(x)}{\lambda a(x)+b(x)} \widetilde{u}(x), \forall \widetilde{u} \in L^{2}\left(E_{2}\right) .
$$

Then $\mathcal{F}$ is a closed operator on $L^{2}\left(E_{2}\right)$. In fact, for any sequence $\left\{\widetilde{u}_{n}\right\} \subset L^{2}\left(E_{2}\right)$, if

$$
\left\|\widetilde{u}_{n}-\widetilde{u}\right\|_{L^{2}\left(E_{2}\right)} \rightarrow 0,\left\|\mathcal{F} \widetilde{u}_{n}-\widehat{u}\right\|_{L^{2}\left(E_{2}\right)} \rightarrow 0
$$

for some $\widetilde{u}, \widehat{u} \in L^{2}\left(E_{2}\right)$, then there exist subsequences $\left\{\widetilde{u}_{n_{k}}\right\}$ and $\left\{\mathcal{F} \widetilde{u}_{n_{k}}\right\}$ converge to $\widetilde{u}$ and $\widehat{u}$ almost everywhere for $x \in E_{2}$, respectively. Therefore, by definition (3.23), we have

$$
(\mathcal{F} \widetilde{u})(x)=\frac{a(x)}{\lambda a(x)+b(x)} \widetilde{u}(x)=\widehat{u}(x), x \in E_{2} \text { a.e.. }
$$


Hence $\mathcal{F}$ is closed on $L^{2}\left(E_{2}\right)$. By the closed graph theorem, $\mathcal{F}$ is bounded on $L^{2}\left(E_{2}\right)$, which implies that

$$
\frac{a}{\lambda a+b} \in L^{\infty}\left(E_{2}\right) \text {. }
$$

This contradicts to $\lambda a(\xi)+b(\xi)=0$ and continuity of $a, b$. Hence $\mathcal{R}(\lambda I-\mathcal{Q}) \neq \mathcal{H}_{1}$. Therefore

$$
\{\lambda \in \mathbb{C} \mid \lambda a(\xi)+b(\xi)=0 \text { for some } \xi \in[0,1]\} \subseteq \sigma(\mathcal{Q}) .
$$

Combining (3.20), (3.22) and (3.24) gives (3.16).

Finally, we show (3.15). Since $\sigma_{\text {ess }}(\mathcal{Q}) \subseteq \sigma(\mathcal{Q})$, we only need to show that $\sigma(\mathcal{Q}) \subseteq \sigma_{\text {ess }}(\mathcal{Q})$. Let

$$
m=\min _{0 \leq x \leq 1}\{\lambda \mid \lambda a(x)+b(x)=0\}, \quad M=\max _{0 \leq x \leq 1}\{\lambda \mid \lambda a(x)+b(x)=0\} .
$$

By (3.16) and (3.22), it suffices to show that $[m, M] \subset \sigma_{\text {ess }}(\mathcal{Q})$. There are two cases:

Case I: $m=M$. In this case, $b(x) / a(x)=-m$ is a constant. It follows from (3.21) that

$$
(m I-\mathcal{Q})(f, g)=(0, m g) .
$$

Hence,

$$
\mathcal{R}(\mathcal{D}) \times\{0\} \subset \mathcal{N}(m I-\mathcal{Q}),
$$

which means, by Definition 3.3, that

$$
\lambda=m \in \sigma_{\text {ess }}(\mathcal{Q}) \text {. }
$$

Case II: $m<M$. In this case, $\lambda$ can be taken as any point of interval $[m, M]$ by the continuity of $b(x) / a(x)$. So by (3.16), $[m, M] \subseteq \sigma(\mathcal{Q})$. Since $\mathcal{Q}$ is self-adjoint, $[m, M] \subseteq \sigma_{\text {ess }}(\mathcal{Q})$ follows from Theorem 5 of [6, p.1395] which says that for a self-adjoint operator, all non-isolated spectrum must be essential spectrum (note that in [6], the essential spectrum of a closed operator is defined as only those that (i) of our Definition 3.1 is satisfied).

With these preparations, we could summarize the properties of $\sigma_{\text {ess }}(\mathcal{A})$ as Theorem 3.8 following.

Theorem 3.8. Let $\mathcal{A}$ be defined by (2.4). Then the following assertions hold.

(i) The essential spectrum of operator $\mathcal{A}$ is given by

$$
\sigma_{\text {ess }}(\mathcal{A})=\{\lambda \in \mathbb{C} \mid a(\xi)+\lambda b(\xi)=0 \text { for some } \xi \in[0,1]\} .
$$

(ii) $\sigma(\mathcal{A}) \backslash \sigma_{\text {ess }}(\mathcal{A})$ consists of at most countable isolated eigenvalues of finite algebraic multiplicity.

Proof. Suppose (i) is valid. Then $\sigma(\mathcal{A}) \backslash \sigma_{\text {ess }}(\mathcal{A})$ is an open connected subset of $\mathbb{C} \backslash \sigma_{\text {ess }}(\mathcal{A})$, (ii) is then a direct consequence of Theorem 2.1 of [8, p.373]. So only proof of (i) is needed. Since $\widetilde{\mathcal{A}}^{-1}=\mathcal{P}+\mathcal{Q}$ and $\mathcal{P}$ is compact, it follows that

$$
\sigma_{\text {ess }}\left(\widetilde{\mathcal{A}}^{-1}\right)=\sigma_{\text {ess }}(\mathcal{Q})=\{0\} \cup\{\lambda \in \mathbb{C} \mid \lambda a(\xi)+b(\xi)=0 \text { for some } \xi \in[0,1]\}
$$

Since $\lambda \in \sigma_{\text {ess }}\left(\widetilde{\mathcal{A}}^{-1}\right)$ if and only if $\lambda^{-1} \in \sigma_{\text {ess }}(\widetilde{\mathcal{A}})$, we have

$$
\sigma_{e s s}(\widetilde{\mathcal{A}})=\{\lambda \in \mathbb{C} \mid a(\xi)+\lambda b(\xi)=0 \text { for some } \xi \in[0,1]\}
$$

The desired result then follows directly through the relation (3.10).

Next, we consider the continuous spectrum for the system (2.1).

Lemma 3.9. Let $\mathcal{A}$ be defined by (2.4) and the following conditions are satisfied:

$$
\left\{\begin{array}{l}
a(x), b(x) \text { and } \rho(x) \text { are analytic in }[0,1] \\
\text { for any } \lambda \in \mathbb{R}, \frac{(x-\xi)^{2}}{a(x)+\lambda b(x)} \text { is analytic in a neighboorhood of any } \xi \in[0,1] .
\end{array}\right.
$$

Then the set of the continuous spectrum $\sigma_{c}(\mathcal{A})$ of $\mathcal{A}$ satisfies

$$
\sigma_{c}(\mathcal{A})=\sigma_{\text {ess }}(\mathcal{A})=\{\lambda \in \mathbb{C} \mid a(\xi)+\lambda b(\xi)=0 \text { for some } \xi \in[0,1]\} .
$$


Proof. Suppose that $a(\xi)+\lambda b(\xi)=0$ for some $\xi \in[0,1], \lambda \in \mathbb{R}$. If $\lambda \in \sigma_{p}(\mathcal{A})$, then there exists a nonzero $f \in H_{0}^{1}(0,1)$ satisfying the characteristic equation (3.1). The proof will be accomplished if we can show that $f \equiv 0$ because $\sigma_{r}(\mathcal{A})=\emptyset$ claimed by Theorem 3.1. This will be divided into three steps:

Step 1: We claim that in a neighborhood of $\xi$,

$$
f(x)=C_{\xi}\left(1+\sum_{n=1}^{\infty} a_{n}(x-\xi)^{n}\right) \text { when } \xi \text { is the first order zero point of } a+\lambda b
$$

or there exists a $r_{1}>0$ such that

$$
f(x)=D_{\xi}(x-\xi)^{r_{1}}\left[1+\sum_{n=1}^{\infty} b_{n}(x-\xi)^{n}\right] \text { when } \xi \text { is the second order zero point of } a+\lambda b
$$

where $C_{\xi}$ and $D_{\xi}$ are constants and each series in Eq. (3.27) and (3.28) converges uniformly in a neighborhood of $\xi$ and defines a function that is analytic at $x=\xi$.

It follows from (3.26) that $\xi$ is the regular singular point of the first equation in (3.1). Using Theorem 4.4 of [3, p.192], (3.1) must admit a Frobenius series solution in a neighborhood of $\xi$. The procedure is as follows. By (3.26), assume that

$$
a(x)+\lambda b(x)=(x-\xi)^{k} \varphi(x),
$$

where $k=1$ or 2 and $\varphi$ is analytic in $[0,1], \varphi(\xi) \neq 0$. Thus,

$$
\frac{(a(x)+\lambda b(x))^{\prime}}{a(x)+\lambda b(x)}=\frac{k}{x-\xi}+\frac{\varphi^{\prime}(x)}{\varphi(x)} \text {. }
$$

Let

$$
p_{0}=\lim _{x \rightarrow \xi}(x-\xi) \frac{(a(x)+\lambda b(x))^{\prime}}{a(x)+\lambda b(x)}, q_{0}=\lim _{x \rightarrow \xi}(x-\xi)^{2} \frac{-\lambda^{2} \rho(x)}{a(x)+\lambda b(x)} .
$$

The indicial equation of (3.1) is (see e.g., Theorem 4.4 of [3, p.192])

$$
F(r)=r(r-1)+p_{0} r+q_{0}=0 .
$$

A simple calculation shows that $p_{0}=1, q_{0}=0$ when $\xi$ is the first order zero point of $a+\lambda b$, and $p_{0}=2, q_{0} \neq 0$ while $\xi$ is the second order zero point of $a+\lambda b$.

Since $f$ is required to be continuous, when $\xi$ is the first order zero point of $a+\lambda b, F(r)=0$ has only zero solution and hence $f$ is of the form (3.27). While $\xi$ is the second order zero point of $a+\lambda b$, let $r_{1}, r_{2}$ be the roots of $F(r)=0$ :

$$
r_{1,2}=\frac{-1 \pm \sqrt{1-4 q_{0}}}{2}
$$

If $r_{1}$ is a nonreal number, then $\operatorname{Re}\left(r_{1}\right)=-\frac{1}{2}$. In this case, $f$ must be identical to zero in a neighborhood of $\xi$ and $D_{\xi}=0$ in (3.28). Otherwise, we may suppose $r_{1}>0>r_{2}$. Since $f$ is continuous in $[0,1]$, it must be of the form (3.28).

Step 2: We claim that there is a sequence $\left\{\gamma_{n}\right\}_{n=1}^{\infty} \subset[0,1], \gamma_{i} \neq \gamma_{j}$ for any $i \neq j, i, j=1,2, \cdots$, such that $f\left(\gamma_{n}\right)=0$ for $n=1,2, \cdots$, and

$$
\lim _{n \rightarrow \infty} \gamma_{n}=\xi
$$

To do this, it suffices to show that for any $\left[x_{1}, x_{2}\right] \subset[0,1]$, if $\xi \in\left[x_{1}, x_{2}\right]$ and $f\left(x_{1}\right)=f\left(x_{2}\right)=0$, then there exists a $\gamma \in\left(x_{1}, x_{2}\right)$ such that $f(\gamma)=0$. In fact, if there exists a second order zero point $\gamma$ of $a+\lambda b$ in $\left(x_{1}, x_{2}\right)$, it follows from Step 1 that

$$
f(\gamma)=0
$$

If there exists no second order zero point of $a+\lambda b$ in $\left(x_{1}, x_{2}\right)$, by Step $\mathbf{1}, f$ must be analytic in $\left(x_{1}, x_{2}\right)$. By Rolle's theorem, it follows that there exits an $\eta \in\left(x_{1}, x_{2}\right)$ such that

$$
f^{\prime}(\eta)=0
$$


If $\xi=\eta$, then by $(3.1)$ and $(a+\lambda b)(\xi)=0$, it has

$$
f(\eta)=0 .
$$

In this case, we take $\gamma=\eta$. If $\xi \neq \eta$, then we have

$$
\left[(a+\lambda b) f^{\prime}\right](\xi)=\left[(a+\lambda b) f^{\prime}\right](\eta)=0 .
$$

By using Rolle's theorem again, there exists a $\gamma$ between $\xi$ and $\eta$ such that

$$
\left[(a+\lambda b) f^{\prime}\right]^{\prime}(\gamma)=0,
$$

which yields $f(\gamma)=0$ from (3.1).

Step 3: It follows from Step 1 and Step 2 that there is a neighborhood $\mathcal{O}_{\xi}$ of $\xi$ such that

$$
f \equiv 0 \text { in } \mathcal{O}_{\xi}
$$

Since $f$ is identical to zero in a neighborhood of any regular singular point $\xi, f$ must be identical to zero everywhere by the uniqueness theorem of the regular ordinary differential equations. The proof is complete.

Theorem 3.10. Let $\mathcal{A}$ be defined by (2.4) and $a(x), b(x), \rho(x)$ are analytic in $[0,1]$. Then

$$
\sigma_{c}(\mathcal{A})=\sigma_{\text {ess }}(\mathcal{A})=\{\lambda \in \mathbb{C} \mid a(\xi)+\lambda b(\xi)=0 \text { for some } \xi \in[0,1]\} .
$$

Proof. Suppose $a(\xi)+\lambda b(\xi)=0$ for some $\xi \in[0,1]$ and $\lambda \in \mathbb{C}$. If $a \equiv-\lambda b$, it is trivially that the solution $f$ of Eq. (3.1) must be identical to zero. By Lemma 3.9, we may assume that

$$
a(x)+\lambda b(x)=(x-\xi)^{m} \varphi(x),
$$

where $m>2$ is a positive integer and $\varphi$ is analytic in $[0,1], \varphi(\xi) \neq 0$. We show that $f \equiv 0$. This case corresponds the irregular singular point for Eq. (3.1). The proof will be divided into three steps:

Step 1: We claim that $f(\xi)=0$. In fact, we can rewrite (3.1) as

$$
f^{\prime \prime}(x)+\frac{1}{x-\xi}\left[m+\frac{\varphi^{\prime}(x)}{\varphi(x)}(x-\xi)\right] f^{\prime}(x)+\frac{1}{(x-\xi)^{m}} \frac{-\lambda^{2} \rho(x)}{\varphi(x)} f(x)=0
$$

with the boundary conditions:

$$
f(0)=f(1)=0 .
$$

By the analyticity of $\varphi$ and $\rho$, we may assume that

$$
\left\{\begin{array}{l}
c(x)=\frac{1}{x-\xi}\left[m+\frac{\varphi^{\prime}(x)}{\varphi(x)}(x-\xi)\right]=\frac{1}{x-\xi}\left[m+\sum_{i=1}^{\infty} h_{i}(x-\xi)^{i}\right], \\
d(x)=\frac{1}{(x-\xi)^{m}} \frac{-\lambda^{2} \rho(x)}{\varphi(x)}=\frac{1}{(x-\xi)^{m}}\left[l_{0}+\sum_{i=1}^{\infty} l_{i}(x-\xi)^{i}\right]
\end{array}\right.
$$

where the two series on the right side above are the Taylor series and by assumption $l_{0} \neq 0$. We only need to discuss the case of $x \geq \xi$ since the case of $x \leq \xi$ can be treated similarly. Let $x-\xi=t^{2}$. Then (3.30) is equivalent to

$$
y^{\prime \prime}(t)+C(t) y^{\prime}(t)+D(t) y(t)=0
$$

where

$$
C(t)=2 t c\left(\xi+t^{2}\right)-\frac{1}{t}=\frac{2 m-1}{t}+2\left[h_{1} t+h_{2} t^{3}+\cdots+h_{n} t^{2 n+1}+\cdots\right]
$$

and

$$
D(t)=4 t^{2} d\left(\xi+t^{2}\right)=\frac{4}{t^{2 m-2}}\left[l_{0}+l_{1} t^{2}+l_{2} t^{4}+\cdots+l_{n} t^{2 n}+\cdots\right]
$$


Then $f(\xi)=0$ is equivalent to $y(0)=0$. We choose $k=m-2$ and let

$$
c_{0}=\left.t^{k+1} C(t)\right|_{t=0}=0, \quad d_{0}=\left.t^{2 k+2} D(t)\right|_{t=0}=4 l_{0} .
$$

Then the solutions of (3.31) are of the form (see, e.g., [5, p.224]).

$$
y(t)=\mathrm{e}^{F(t)} Y(t),
$$

where

$$
F(t)=\frac{A_{k}}{t^{k}}+\frac{A_{k-1}}{t^{k-1}}+\cdots+\frac{A_{1}}{t}
$$

and

$$
Y(t)=\sum_{n=0}^{\infty} a_{n} t^{n+s}, a_{0} \neq 0
$$

is a Frobenius series. Substitute (3.32) into (3.31) to obtain the differential equation satisfied by $Y$ :

$$
Y^{\prime \prime}(t)+\left[C(t)+2 F^{\prime}(t)\right] Y^{\prime}(t)+\left[D(t)+C(t) F^{\prime}(t)+\left[F^{\prime}(t)\right]^{2}+F^{\prime \prime}(t)\right] Y(t)=0
$$

Choose the constants $A_{n}, n=1,2, \cdots, k$ to eliminate the most singular terms in the coefficient of $Y$ in (3.35) to get, after a calculation, that

$$
A_{k}=\frac{c_{0} \pm \sqrt{c_{0}^{2}-4 d_{0}}}{2 k}= \pm \frac{2}{k} \sqrt{-l_{0}} \neq 0, A_{k-1}=A_{k-3}=\cdots=0 .
$$

There are two cases:

Case I: $l_{0}>0$. In this case, $\operatorname{Re}\left(A_{k}\right)=0$. By a simple calculation, we find that $\operatorname{Re}\left(A_{n}\right)=0, n=1,2, \cdots, k$, and by Eq. (6.53) of [5, p.226],

$$
\operatorname{Re}(s)=-\frac{-(2 m-1) k A_{k}+k(k+1) A_{k}}{-2 k A_{k}}=-\frac{m}{2}<0 .
$$

Let

$$
F(t)=i \tau(t), Y(t)=t^{s}[u(t)+i v(t)], s=\alpha+i \beta,
$$

where $\alpha=-\frac{m}{2}, \beta, \tau(t), u(t), v(t) \in \mathbb{R}$ and $u, v$ are analytic at $t=0, u^{2}(0)+v^{2}(0) \neq 0$. Then

$$
\begin{aligned}
y(t)= & {[\cos \tau(t)+i \sin \tau(t)] t^{\alpha}[\cos (\beta \ln t)+i \sin (\beta \ln t)][u(t)+i v(t)] } \\
= & t^{\alpha}[\cos (\tau(t)+\beta \ln t)+i \sin (\tau(t)+\beta \ln t)][u(t)+i v(t)] \\
= & t^{\alpha}[u(t) \cos (\tau(t)+\beta \ln t)-v(t) \sin (\tau(t)+\beta \ln t)] \\
& +i t^{\alpha}[v(t) \cos (\tau(t)+\beta \ln t)+u(t) \sin (\tau(t)+\beta \ln t)] .
\end{aligned}
$$

Let $z(t)=\tau(t)+\beta \ln t$. Since $A_{k} \neq 0, \lim _{t \rightarrow 0^{+}} z(t)=\infty$, this together with the continuity of $z(t)$ enables us to easily show that $[u(t) \cos z(t)-v(t) \sin z(t)]$ and $[v(t) \cos z(t)+u(t) \sin z(t)]$ are linearly independent. Therefore the general solution of (3.31) is of the form

$$
y(t)=t^{\alpha}\left\{b_{1}[u(t) \cos z(t)-v(t) \sin z(t)]+b_{2}[v(t) \cos z(t)+u(t) \sin z(t)]\right\},
$$

where $b_{1}$ and $b_{2}$ are real constants. Since $f(x)$ is continuous at $x=\xi$, so is for $y(t)$ at $t=0$. Since $\alpha<0$, it must have

$$
\lim _{t \rightarrow 0^{+}}\left\{b_{1}[u(t) \cos z(t)-v(t) \sin z(t)]+b_{2}[v(t) \cos z(t)+u(t) \sin z(t)]\right\}=0 .
$$

Since $\lim _{t \rightarrow 0^{+}} z(t)=\infty$, one can find two positive sequences $\left\{t_{n_{1}}\right\}$ and $\left\{t_{n_{2}}\right\}$ such that

$$
\lim _{n_{1} \rightarrow \infty} t_{n_{1}}=0, \lim _{n_{2} \rightarrow \infty} t_{n_{2}}=0,
$$




$$
\cos z\left(t_{n_{1}}\right)=1, \quad \sin z\left(t_{n_{1}}\right)=0, \quad \cos z\left(t_{n_{2}}\right)=0, \quad \sin z\left(t_{n_{2}}\right)=1 .
$$

This together with (3.37) gives

$$
\left\{\begin{array}{l}
b_{1} u(0)+b_{2} v(0)=\lim _{n_{1} \rightarrow \infty}\left[b_{1} u\left(t_{n_{1}}\right)+b_{2} v\left(t_{n_{1}}\right)\right]=0, \\
-b_{1} v(0)+b_{2} u(0)=\lim _{n_{2} \rightarrow \infty}\left[-b_{1} v\left(t_{n_{2}}\right)+b_{2} u\left(t_{n_{2}}\right)\right]=0 .
\end{array}\right.
$$

Since $u^{2}(0)+v^{2}(0) \neq 0$, it has $b_{1}=b_{2}=0$. By (3.36),

$$
y(t) \equiv 0 \text {. }
$$

Case II: $l_{0}<0$. In this case, $A_{k} \in \mathbb{R}$. And by the similar calculation as Case I, we have $A_{n} \in \mathbb{R}, n=1,2, \cdots, k$ and the general solution of (3.31) is of the form

$$
y(t)=c_{1} \mathrm{e}^{F(t)} Y_{1}(t)+c_{2} \mathrm{e}^{-F(t)} Y_{2}(t)
$$

where $c_{1}$ and $c_{2}$ are constants, $F(t)$ is of the form (3.33), and $Y_{i}(t), i=1,2$ is of the form (3.34). Now, we may assume without loss of generality that $A_{k}=-\frac{2}{k} \sqrt{-l_{0}}<0$. Then by the continuity of $y$ and $\lim _{t \rightarrow 0^{+}} \mathrm{e}^{-F(t)} Y_{2}(t)=\infty$, it must have

$$
y(t)=c_{1} \mathrm{e}^{F(t)} Y_{1}(t)
$$

and hence $y(0)=0$.

Step 2: We claim that there is a sequence $\left\{\gamma_{n}\right\}_{n=1}^{\infty} \subset[0,1], \gamma_{i} \neq \gamma_{j}$ for any $i \neq j, i, j=1,2, \cdots$, such that $f\left(\gamma_{n}\right)=0$ for $n=1,2, \cdots$, and

$$
\lim _{n \rightarrow \infty} \gamma_{n}=\xi
$$

To do this, it suffices to show that for any $\left[x_{1}, x_{2}\right] \subset[0,1]$, if $\xi \in\left[x_{1}, x_{2}\right]$ and $f\left(x_{1}\right)=f\left(x_{2}\right)=0$, then there exists a $\gamma \in\left(x_{1}, x_{2}\right)$ such that $f(\gamma)=0$. In fact, if there exists a zero point $\gamma$ of $a+\lambda b$ in $\left(x_{1}, x_{2}\right)$ whose order is greater than one, it follows from Step 1 and the proof of Lemma 3.9 that

$$
f(\gamma)=0
$$

Otherwise, by the proof of Lemma 3.9, $f$ is analytic at any first order zero of $a+\lambda b$ in $\left(x_{1}, x_{2}\right)$, and $\xi=x_{1}$ or $\xi=x_{2}$. But since the solution $y$ of (3.31) is of (3.38) or (3.39), it is differentiable in a neighborhood of $t=0$ except $t=0$. So the solution $f$ of (3.1) is differentiable in a neighborhood of $x=\xi$ except $x=\xi$. In any case, $f$ is differentiable in $\left(x_{1}, x_{2}\right)$. By Rolle's theorem, there exits an $\eta \in\left(x_{1}, x_{2}\right)$ such that

$$
f^{\prime}(\eta)=0
$$

Clearly $\xi \neq \eta$. Since

$$
\left[(a+\lambda b) f^{\prime}\right](\xi)=\left[(a+\lambda b) f^{\prime}\right](\eta)=0,
$$

using Rolle's theorem again, there exists a $\gamma$ between $\xi$ and $\eta$ such that

$$
\left[(a+\lambda b) f^{\prime}\right]^{\prime}(\gamma)=0,
$$

which yields $f(\gamma)=0$ from (3.1).

Step 3: By Step 2, the solution $y$ of (3.31) has infinitely many zero points approaching zero. Since $y$ is of (3.38) or (3.39), $y \equiv 0$ in a neighborhood of $t=0$. Equivalently, there is a neighborhood $\mathcal{O}_{\xi}$ of $\xi$ such that

$$
f \equiv 0 \text { in } \mathcal{O}_{\xi}
$$

Since there are at most finite number of singular points $\xi, f$ must be identical to zero everywhere by the uniqueness of the solution for regular linear ordinary differential equations. The proof is complete. 
If there is no analyticity, Theorem 3.10 is not true anymore. This is suggested by many studies on Sturm-Liouville problem. The following is an counter-example.

Example 3.11. Let

$$
-p(x)=a(x)+\lambda b(x)=-x^{1 / 3} .
$$

Then $1 / p \in L^{1}(0,1)$. According to Theorem 0 of [7], there are countable number of positive $\mu$ such that the Sturm-Liouville problem:

$$
-\left(p(x) f^{\prime}(x)\right)^{\prime}=\mu f(x), x \in(0,1), f(0)=f(1)=0
$$

admits nonzero absolutely continuous solutions $f$. Take specially $\mu>0$ for such a $\mu$. Then we may choose

$$
a(x)=\sqrt{\mu}+2 x^{1 / 3}, b(x)=1+\frac{3}{\sqrt{\mu}} x^{1 / 3}, \lambda=-\sqrt{\mu}, \rho(x)=1 .
$$

Eq. (3.1) is now having a nonzero absolutely continuous solution $f$. Suppose $\left(p f^{\prime}\right)(0)=c$. We show that $f \in H_{0}^{1}(0,1)$ or equivalently $f^{\prime} \in L^{2}(0,1)$, which hence severs as a counter-example. Indeed, set

$$
\alpha=\frac{1}{3}, A(x)=\left(\begin{array}{cc}
0 & x^{-\alpha} \\
-\mu & 0
\end{array}\right), y(x)=\left(\begin{array}{c}
f(x) \\
p(x) f^{\prime}(x)
\end{array}\right), y_{0}=y(0)=\left(\begin{array}{c}
0 \\
c
\end{array}\right) .
$$

Then (3.41) is rewritten as

$$
\frac{d y}{d x}=A(x) y(x)
$$

According to Theorem 1 of [19, Sect. 16], the above equation is equivalent to the following integral equation

$$
y(x)=y_{0}+\int_{0}^{x} A(\xi) y(\xi) d \xi
$$

for which the solution can be represented uniformly in $[0,1]$ as

$$
y(x)=\lim _{n \rightarrow \infty} y_{n}(x),
$$

where

$$
y_{n+1}(x)=\left(\begin{array}{c}
f_{n+1}(x) \\
h_{n+1}(x)
\end{array}\right)=y_{0}+\int_{0}^{x} A(\xi) y_{n}(\xi) d \xi, n=0,1,2, \cdots
$$

A direct computation shows that

$$
\left\{\begin{array}{l}
f_{2 n+1}(x)=f_{2 n+2}(x)=\frac{c}{1-\alpha} x^{1-\alpha}+\sum_{k=1}^{n} a_{k} x^{k+(k+1)(1-\alpha)}, \\
h_{2 n}(x)=h_{2 n+1}(x)=x^{\alpha} f_{2 n+1}^{\prime}(x),
\end{array}\right.
$$

where

$$
a_{k}=\frac{(-1)^{k} c \mu^{k}}{(1-\alpha)(2-\alpha)(3-2 \alpha)(4-2 \alpha)(5-3 \alpha) \cdots(2 k-k \alpha)(2 k+1-(k+1) \alpha)} .
$$

Therefore,

$$
f(x)=\frac{c}{1-\alpha} x^{1-\alpha}+\sum_{k=1}^{\infty} a_{k} x^{k+(k+1)(1-\alpha)} .
$$


The above series is absolutely uniformly convergent since $|f(x)| \leq|c| /(1-\alpha)+\sum_{k=1}^{\infty}\left|a_{k}\right|$ and

$$
\lim _{k \rightarrow \infty} \frac{\left|a_{k}\right|}{\left|a_{k-1}\right|}=\lim _{k \rightarrow \infty} \frac{\mu}{(2 k-k \alpha)(2 k+1-(k+1) \alpha)}=0 .
$$

Now,

$$
f^{\prime}(x)=c x^{-\alpha}+\sum_{k=1}^{\infty} a_{k}[2 k+1-(k+1) \alpha] x^{k-1+(k+1)(1-\alpha)} .
$$

The above series is also absolutely uniformly convergent since

$$
\left|\sum_{k=1}^{\infty} a_{k}[2 k+1-(k+1) \alpha] x^{k-1+(k+1)(1-\alpha)}\right| \leq \sum_{k=1}^{\infty}\left|a_{k}[2 k+1-(k+1) \alpha]\right|
$$

and

$$
\lim _{k \rightarrow \infty} \frac{\left|a_{k}[2 k+1-(k+1) \alpha]\right|}{\left|a_{k-1}[2 k-1-k \alpha]\right|}=\lim _{k \rightarrow \infty} \frac{\mu}{(2 k-k \alpha)(2 k-1-k \alpha)}=0 .
$$

This shows that $f^{\prime}(x)=c x^{-\alpha}+g(x)$ where $g$ is a continuous function. Hence $f^{\prime} \in L^{2}(0,1)$.

\section{Asymptotic behavior of eigenvalues}

In this section, we consider the asymptotic behavior of eigenvalues for the system (2.1). To do this, we assume further that

$$
a(x), b(x) \in C^{1}[0,1] \text { and } a(x), b(x)>0 \text { for all } x \in[0,1] .
$$

Suppose that $\lambda$ is an eigenvalue with large modulus. Then

$$
a(x)+\lambda b(x) \neq 0 \text { for any } x \in[0,1]
$$

and we rewrite the characteristic equation (3.1) as

$$
\left\{\begin{array}{l}
{[a(x)+\lambda b(x)] f^{\prime \prime}(x)+\left[a^{\prime}(x)+\lambda b^{\prime}(x)\right] f^{\prime}(x)=\lambda^{2} \rho(x) f(x),} \\
f(0)=f(1)=0 .
\end{array}\right.
$$

By (4.3), it is apparently seen that $\lambda$ must be geometrically simple.

The following Lemma 4.1 is immediate.

Lemma 4.1. Let $\lambda \in \mathbb{C}$. Then as $|\lambda| \rightarrow \infty$, it has

$$
\frac{1}{a(x)+\lambda b(x)}=\frac{1}{\lambda b(x)} \cdot \frac{1}{1+\frac{a(x)}{\lambda b(x)}}=\frac{1}{\lambda b(x)}-\frac{a(x)}{\lambda^{2} b^{2}(x)}+\frac{a^{2}(x)}{\lambda^{3} b^{3}(x)}+\mathcal{O}\left(|\lambda|^{-4}\right)
$$

and

$$
[a(x)+\lambda b(x)] f^{\prime \prime}(x)+\left[a^{\prime}(x)+\lambda b^{\prime}(x)\right] f^{\prime}(x)=\lambda^{2} \rho(x) f(x)
$$

has the following asymptotic expression:

$$
\begin{aligned}
f^{\prime \prime}(x) & +\left[\frac{1}{\lambda b(x)}-\frac{a(x)}{\lambda^{2} b^{2}(x)}+\frac{a^{2}(x)}{\lambda^{3} b^{3}(x)}+\mathcal{O}\left(|\lambda|^{-4}\right)\right]\left[a^{\prime}(x)+\lambda b^{\prime}(x)\right] f^{\prime}(x) \\
& -\lambda\left[1-\frac{a(x)}{\lambda b(x)}+\frac{a^{2}(x)}{\lambda^{2} b^{2}(x)}+\mathcal{O}\left(|\lambda|^{-3}\right)\right] \rho_{0}^{2}(x) f(x)=0 .
\end{aligned}
$$

where

$$
\rho_{0}(x)=\sqrt{\frac{\rho(x)}{b(x)}}
$$


In order to find the asymptotic fundamental solutions of (4.6), we introduce the following space-scaling transformation:

$$
\phi(z)=f(x), z=\frac{1}{h} \int_{0}^{x} \rho_{0}(\tau) d \tau, h=\int_{0}^{1} \rho_{0}(\tau) d \tau .
$$

Under this transformation, (4.6) becomes

$$
\begin{aligned}
\phi^{\prime \prime}(z) & +h\left\{\frac{\rho_{0}^{\prime}(x)}{\rho_{0}^{2}(x)}+\frac{1}{\rho_{0}(x)}\left[\frac{1}{\lambda b(x)}-\frac{a(x)}{\lambda^{2} b^{2}(x)}+\frac{a^{2}(x)}{\lambda^{3} b^{3}(x)}+\mathcal{O}\left(|\lambda|^{-4}\right)\right]\left[a^{\prime}(x)+\lambda b^{\prime}(x)\right]\right\} \phi^{\prime}(z) \\
& -\lambda h^{2}\left[1-\frac{a(x)}{\lambda b(x)}+\frac{a^{2}(x)}{\lambda^{2} b^{2}(x)}+\mathcal{O}\left(|\lambda|^{-3}\right)\right] \phi(z)=0
\end{aligned}
$$

with the boundary conditions:

$$
\phi(0)=\phi(1)=0
$$

Proposition 4.2. The equation (4.6) with boundary condition (4.3) is equivalent to (4.9) and (4.10). That is, ( $\lambda$, $f$ ), $f \neq 0$, satisfies (4.6) and boundary conditions (4.3) if and only if $(\lambda, \phi), \phi \neq 0$, satisfies (4.9) and (4.10).

Now we consider (4.9) and (4.10). Since the eigenvalues are symmetric about the real axis and $\operatorname{Re} \lambda \leq 0$ for any $\lambda \in \sigma(\mathcal{A})$, we only consider those eigenvalues $\lambda$ with $\frac{\pi}{2} \leq \arg \lambda \leq \pi$.

Let $\lambda=\mu^{2}$. Then as $\frac{\pi}{2} \leq \arg \lambda \leq \pi$, we consider $\mu$ locating on the following sector:

$$
\mathcal{S}=\left\{\mu \in \mathbb{C} \mid \frac{\pi}{4} \leq \arg \mu \leq \frac{\pi}{2}\right\}
$$

Lemma 4.3. Suppose $\lambda=\mu^{2} \neq 0$. Then for $z \in[0,1]$ and $\mu \in \mathcal{S}$,

$$
\mathrm{e}^{\mu h z}, \mathrm{e}^{-\mu h z}
$$

are linearly independent fundamental solutions of

$$
\phi^{\prime \prime}(z)-\mu^{2} h^{2} \phi(z)=0
$$

and for $|\mu|$ large enough, (4.9) has the following asymptotic fundamental solutions:

$$
\left\{\begin{array}{l}
\phi_{1}(z)=\mathrm{e}^{\mu h z}\left[\phi_{10}(z)+\phi_{11}(z) \mu^{-1}+\mathcal{O}\left(\mu^{-2}\right)\right], \\
\phi_{2}(z)=\mathrm{e}^{-\mu h z}\left[\phi_{20}(z)+\phi_{21}(z) \mu^{-1}+\mathcal{O}\left(\mu^{-2}\right)\right]
\end{array}\right.
$$

where

$$
\left\{\begin{array}{l}
\phi_{10}(z)=\phi_{20}(z)=\exp \left\{-\frac{1}{2} \int_{0}^{z} \rho_{1}(x) \rho_{0}(x) d x\right\} \\
\phi_{11}(z)=-\frac{1}{2} \int_{0}^{z} \exp \left\{-\frac{1}{2} \int_{0}^{z-\zeta} \rho_{1}(x) \rho_{0}(x) d x\right\} \rho_{2}(\zeta) d \zeta \\
\phi_{21}(z)=-\phi_{11}(z)=\frac{1}{2} \int_{0}^{z} \exp \left\{-\frac{1}{2} \int_{0}^{z-\zeta} \rho_{1}(x) \rho_{0}(x) d x\right\} \rho_{2}(\zeta) d \zeta
\end{array}\right.
$$

and

$$
\left\{\begin{array}{l}
\rho_{1}(x)=\frac{\rho_{0}^{\prime}(x)}{\rho_{0}^{2}(x)}+\frac{1}{\rho_{0}(x)} \frac{b^{\prime}(x)}{b(x)} \\
\rho_{2}(z)=\frac{1}{h} \phi_{10}^{\prime \prime}(z)+h \frac{a(x(z))}{b(x(z))} \phi_{10}(z)+\left(\frac{\rho_{0}^{\prime}(x(z))}{\rho_{0}^{2}(x(z))}+\frac{1}{\rho_{0}(x(z))} \frac{b^{\prime}(x(z))}{b(x(z))}\right) \phi_{10}^{\prime}(z)
\end{array}\right.
$$


Pro of. The first claim is trivial. We only need to show that (4.13) are the asymptotic fundamental solutions of (4.9). This can be done along the same way of [2] and [19, Sect. 4]. Here we present briefly a simple calculation to (4.13)-(4.15).

Let

$$
\left\{\begin{array}{l}
\widetilde{\phi}_{1}(z, \mu)=\mathrm{e}^{\mu h z}\left[\phi_{10}(z)+\phi_{11}(z) \mu^{-1}\right], \\
\widetilde{\phi}_{2}(z, \mu)=\mathrm{e}^{-\mu h z}\left[\phi_{20}(z)+\phi_{21}(z) \mu^{-1}\right],
\end{array}\right.
$$

where $\phi_{k i}(z), k=1,2, i=0,1$ are some functions to be determined, and

$$
\begin{aligned}
D(\phi)= & \phi^{\prime \prime}(z)-\mu^{2} h^{2}\left[1-\frac{a(x)}{\mu^{2} b(x)}+\frac{a^{2}(x)}{\mu^{4} b^{2}(x)}+\mathcal{O}\left(|\mu|^{-6}\right)\right] \phi(z) \\
& +h\left\{\frac{\rho_{0}^{\prime}(x)}{\rho_{0}^{2}(x)}+\frac{1}{\rho_{0}(x)}\left[\frac{1}{\mu^{2} b(x)}-\frac{a(x)}{\mu^{4} b^{2}(x)}+\frac{a^{2}(x)}{\mu^{6} b^{3}(x)}+\mathcal{O}\left(|\mu|^{-8}\right)\right]\left[a^{\prime}(x)+\mu^{2} b^{\prime}(x)\right]\right\} \phi^{\prime}(z) .
\end{aligned}
$$

Substitute $\widetilde{\phi}_{1}(z, \mu)$ into $D(\phi)$ to yield

$$
\begin{array}{rl}
\mathrm{e}^{-\mu h z} & D\left(\widetilde{\phi}_{1}(z, \mu)\right) \\
= & \mu^{2} h^{2}\left[\phi_{10}(z)+\phi_{11}(z) \mu^{-1}\right]+2 \mu h\left[\phi_{10}^{\prime}(z)+\phi_{11}^{\prime}(z) \mu^{-1}\right]+\left[\phi_{10}^{\prime \prime}(z)+\phi_{11}^{\prime \prime}(z) \mu^{-1}\right] \\
& -\mu^{2} h^{2}\left[1-\frac{a(x)}{\mu^{2} b(x)}+\frac{a^{2}(x)}{\mu^{4} b^{2}(x)}+\mathcal{O}\left(|\mu|^{-6}\right)\right]\left[\phi_{10}(z)+\phi_{11}(z) \mu^{-1}\right] \\
& +h\left\{\frac{\rho_{0}^{\prime}(x)}{\rho_{0}^{2}(x)}+\frac{1}{\rho_{0}(x)}\left[\frac{1}{\mu^{2} b(x)}-\frac{a(x)}{\mu^{4} b^{2}(x)}+\frac{a^{2}(x)}{\mu^{6} b^{3}(x)}+\mathcal{O}\left(|\mu|^{-8}\right)\right]\left[a^{\prime}(x)+\mu^{2} b^{\prime}(x)\right]\right\} \\
& \times\left\{\mu h\left[\phi_{10}(z)+\phi_{11}(z) \mu^{-1}\right]+\left[\phi_{10}^{\prime}(z)+\phi_{11}^{\prime}(z) \mu^{-1}\right]\right\} \\
= & \mu\left[2 h \phi_{10}^{\prime}(z)+h^{2}\left(\frac{\rho_{0}^{\prime}(x)}{\rho_{0}^{2}(x)}+\frac{1}{\rho_{0}(x)} \frac{b^{\prime}(x)}{b(x)}\right) \phi_{10}(z)\right] \\
& +\left[2 h \phi_{11}^{\prime}(z)+h^{2}\left(\frac{\rho_{0}^{\prime}(x)}{\rho_{0}^{2}(x)}+\frac{1}{\rho_{0}(x)} \frac{b^{\prime}(x)}{b(x)}\right) \phi_{11}(z)\right. \\
& \left.+\phi_{10}^{\prime \prime}(z)+h^{2} \frac{a(x)}{b(x)} \phi_{10}(z)+h\left(\frac{\rho_{0}^{\prime}(x)}{\rho_{0}^{2}(x)}+\frac{1}{\rho_{0}(x)} \frac{b^{\prime}(x)}{b(x)}\right) \phi_{10}^{\prime}(z)\right]+\mathcal{O}\left(\mu^{-1}\right) .
\end{array}
$$

Letting the coefficients of $\mu^{1}$ and $\mu^{0}$ be zero gives

$$
2 \phi_{10}^{\prime}(z)+h\left(\frac{\rho_{0}^{\prime}(x)}{\rho_{0}^{2}(x)}+\frac{1}{\rho_{0}(x)} \frac{b^{\prime}(x)}{b(x)}\right) \phi_{10}(z)=0
$$

and

$$
\begin{aligned}
2 h \phi_{11}^{\prime}(z) & +h^{2}\left(\frac{\rho_{0}^{\prime}(x)}{\rho_{0}^{2}(x)}+\frac{1}{\rho_{0}(x)} \frac{b^{\prime}(x)}{b(x)}\right) \phi_{11}(z) \\
& +\phi_{10}^{\prime \prime}(z)+h^{2} \frac{a(x)}{b(x)} \phi_{10}(z)+h\left(\frac{\rho_{0}^{\prime}(x)}{\rho_{0}^{2}(x)}+\frac{1}{\rho_{0}(x)} \frac{b^{\prime}(x)}{b(x)}\right) \phi_{10}^{\prime}(z)=0 .
\end{aligned}
$$

Use the conditions $\phi_{10}(0)=1$ and $\phi_{11}(0)=0$ to obtain

$$
\phi_{10}(z)=\exp \left\{-\frac{1}{2} h \int_{0}^{z} \rho_{1}(x(\tau)) d \tau\right\}
$$

and

$$
\phi_{11}(z)=-\frac{1}{2} \int_{0}^{z} \exp \left\{-\frac{1}{2} h \int_{0}^{z-\zeta} \rho_{1}(x(\tau)) d \tau\right\} \rho_{2}(\zeta) d \zeta
$$


where $\rho_{1}(x)$ and $\rho_{2}(z)$ are given by (4.15). From (4.8), we have

$$
\frac{d z}{d x}=\frac{1}{h} \rho_{0}(x)
$$

and so

$$
\frac{d x}{d z}=\frac{h}{\rho_{0}(x)}
$$

Hence

$$
\phi_{10}(z)=\exp \left\{-\frac{1}{2} \int_{0}^{z} \rho_{1}(x(\tau)) \rho_{0}(x(\tau)) \frac{d x}{d \tau} d \tau\right\}=\exp \left\{-\frac{1}{2} \int_{0}^{z} \rho_{1}(x) \rho_{0}(x) d x\right\}
$$

and

$$
\phi_{11}(z)=-\frac{1}{2} \int_{0}^{z} \exp \left\{-\frac{1}{2} \int_{0}^{z-\zeta} \rho_{1}(x) \rho_{0}(x) d x\right\} \rho_{2}(\zeta) d \zeta
$$

Same arguments also give $\phi_{20}(z)$ and $\phi_{21}(z)$ as in (4.14) and (4.15). The proof is complete.

Let $\lambda=\mu^{2}$ with large modulus and $\mu \in \mathcal{S}$ defined by (4.11). Let $\phi$ be a solution of (4.9) and (4.10). There are constants $c_{1}$ and $c_{2}$ such that

$$
\phi(z)=c_{1} \phi_{1}(z)+c_{2} \phi_{2}(z)
$$

where $\phi_{1}(z)$ and $\phi_{2}(z)$ are fundamental solutions given by (4.13)-(4.15). By using the boundary conditions of (4.10), we have

$$
\Delta(\mu)\left[c_{1}, c_{2}\right]^{\top}=0
$$

where

$$
\Delta(\mu)=\left[\begin{array}{cc}
1 & 1 \\
\phi_{1}(1) & \phi_{2}(1)
\end{array}\right]
$$

Hence, $\phi(z)$ has a nontrivial solution if and only if $\operatorname{det}(\Delta(\mu))=0$, that is, $\mu \in \mathcal{S}$ satisfies the characteristic equation:

$$
\begin{aligned}
\operatorname{det}(\Delta(\mu)) & =\phi_{2}(1)-\phi_{1}(1) \\
& =\mathrm{e}^{-\mu h}\left[\phi_{20}(1)+\phi_{21}(1) \mu^{-1}+\mathcal{O}\left(\mu^{-2}\right)\right]-\mathrm{e}^{\mu h}\left[\phi_{10}(1)+\phi_{11}(1) \mu^{-1}+\mathcal{O}\left(\mu^{-2}\right)\right] \\
& =\phi_{10}(1)\left\{\mathrm{e}^{-\mu h}-\mathrm{e}^{\mu h}+k_{0} \mu^{-1}\left[\mathrm{e}^{-\mu h}+\mathrm{e}^{\mu h}\right]\right\}+\mathcal{O}\left(\mu^{-2}\right) \\
& =0
\end{aligned}
$$

where $k_{0}$ is a constant satisfying

$$
k_{0}=-\frac{\phi_{11}(1)}{\phi_{10}(1)}=\frac{1}{2} \frac{\int_{0}^{1} \exp \left\{-\frac{1}{2} \int_{0}^{1-\zeta} \rho_{1}(x) \rho_{0}(x) d x\right\} \rho_{2}(\zeta) d \zeta}{\exp \left\{-\frac{1}{2} \int_{0}^{1} \rho_{1}(x) \rho_{0}(x) d x\right\}} .
$$

Lemma 4.4. Let $\Delta(\mu)$ be given by (4.19). Then the characteristic determinant $\operatorname{det}(\Delta(\mu))$ has the following asymptotic expression:

$$
\frac{1}{\phi_{10}(1)} \operatorname{det}(\Delta(\mu))=\mathrm{e}^{-\mu h}-\mathrm{e}^{\mu h}+k_{0} \mu^{-1}\left[\mathrm{e}^{-\mu h}+\mathrm{e}^{\mu h}\right]+\mathcal{O}\left(\mu^{-2}\right),
$$

where $k_{0}$ is given by (4.21). 
Theorem 4.5. Let $\lambda=\mu^{2}$ with large modulus and $\mu \in \mathcal{S}$ defined by (4.11). Then $\lambda$, which must be geometrically simple as indicated in the beginning of the section, has the following asymptotic form:

$$
\lambda_{n}=-\frac{n^{2} \pi^{2}}{h^{2}}+2 \frac{k_{0}}{h}+\mathcal{O}\left(n^{-1}\right), \quad n=N, N+1, \ldots,
$$

where $k_{0}$ is given by (4.21) and $h$ is given by (4.8).

Proof. Since in sector $\mathcal{S}, \operatorname{det}(\Delta(\mu))$ has the asymptotic form given by (4.22), by $\operatorname{det}(\Delta(\mu))=0$, we have

$$
\mathrm{e}^{-\mu h}-\mathrm{e}^{\mu h}+k_{0} \mu^{-1}\left[\mathrm{e}^{-\mu h}+\mathrm{e}^{\mu h}\right]+\mathcal{O}\left(\mu^{-2}\right)=0,
$$

which can also be rewritten as

$$
1-\mathrm{e}^{-2 \mu h}+\mathcal{O}\left(\mu^{-1}\right)=0 .
$$

Since in sector $\mathcal{S}$, the solutions of $1-\mathrm{e}^{-2 \mu h}=0$ are given by

$$
\widetilde{\mu}_{n}=\frac{n \pi i}{h}, \quad n=0,1,2, \ldots
$$

it follows from Rouché's theorem that the solutions of Eq. (4.25) have the form of

$$
\mu_{n}=\widetilde{\mu}_{n}+\alpha_{n}=\frac{n \pi i}{h}+\alpha_{n}, \quad \alpha_{n}=\mathcal{O}\left(n^{-1}\right), n=N, N+1, N+2, \ldots
$$

where $N$ is a sufficiently large positive integer. Substitute $\mu_{n}$ into (4.24) and use the fact that $\mathrm{e}^{2 \widetilde{\mu}_{n} h}=1$ to obtain

$$
1-\mathrm{e}^{2 \alpha_{n} h}+k_{0} \mu_{n}^{-1}\left[1+\mathrm{e}^{2 \alpha_{n} h}\right]+\mathcal{O}\left(\mu^{-2}\right)=0
$$

Expand the exponential function in (4.26) according to its Taylor series, to give

$$
2 \alpha_{n} h=2 k_{0} \widetilde{\mu}_{n}^{-1}+\mathcal{O}\left(\mu_{n}^{-2}\right)
$$

Hence we obtain that

$$
\alpha_{n}=\frac{k_{0}}{h} \widetilde{\mu}_{n}^{-1}+\mathcal{O}\left(n^{-2}\right)
$$

and

$$
\mu_{n}=\widetilde{\mu}_{n}+\alpha_{n}=\frac{n \pi i}{h}+\frac{k_{0}}{h} \widetilde{\mu}_{n}^{-1}+\mathcal{O}\left(n^{-2}\right), \quad n=N, N+1, \ldots
$$

Due to $\lambda_{n}=\mu_{n}^{2}$, we get eventually

$$
\lambda_{n}=\left(\frac{n \pi i}{h}\right)^{2}+2 \frac{k_{0}}{h}+\mathcal{O}\left(n^{-1}\right)=-\frac{n^{2} \pi^{2}}{h^{2}}+2 \frac{k_{0}}{h}+\mathcal{O}\left(n^{-1}\right), \quad n=N, N+1, \ldots
$$

Theorem 4.5 is about the asymptotic expression for high eigenfrequencies. To end this section, we indicate that the high eigenfrequencies are actually real, which is stronger than that claimed by Theorem 1 of [20] under the assumption $b>0$.

Proposition 4.6. Suppose $b>0$. Let $\mathcal{A}$ be defined by (2.4) and

$$
\Lambda_{0}=\{\lambda \in \sigma(\mathcal{A}) \mid \operatorname{Im} \lambda \neq 0\} .
$$

Then $\Lambda_{0}$ is a bounded set of $\mathbb{C}$. Moreover, there is no spectrum on the imaginary axis and hence $\operatorname{Re} \lambda \leq-\alpha$ for some $\alpha>0$ for all $\lambda \in \sigma(\mathcal{A})$. 
Proof. By Theorems 3.1 and 3.8, $\Lambda_{0} \subset \sigma_{p}(\mathcal{A})$. For any $\lambda=\tau+i \omega \in \Lambda_{0}$, we may take $(f, \lambda f), f \neq 0$ to be an eigenfunction corresponding to $\lambda$. Multiply the first equation of (3.1) by $\overline{f_{n}}$ and then integrate over $[0,1]$ with respect to $x$, to obtain, after separating real part and imaginary part, that

$$
\left\{\begin{array}{l}
\left(\tau^{2}-\omega^{2}\right) \int_{0}^{1} \rho(x)|f(x)|^{2} d x+\int_{0}^{1}[a(x)+\tau b(x)]\left|f^{\prime}(x)\right|^{2} d x=0, \\
2 \tau \omega \int_{0}^{1} \rho(x)|f(x)|^{2} d x+\omega \int_{0}^{1} b(x)\left|f^{\prime}(x)\right|^{2} d x=0,
\end{array}\right.
$$

which is equivalent to

$$
\left\{\begin{array}{l}
|\lambda|^{2} \int_{0}^{1} \rho(x)|f(x)|^{2} d x=\int_{0}^{1} a(x)\left|f^{\prime}(x)\right|^{2} d x \\
-2 \operatorname{Re} \lambda \int_{0}^{1} \rho(x)|f(x)|^{2} d x=\int_{0}^{1} b(x)\left|f^{\prime}(x)\right|^{2} d x .
\end{array}\right.
$$

Thus $\operatorname{Re} \lambda \neq 0$, and

$$
|\lambda| \leq \frac{|\lambda|^{2}}{|\operatorname{Re} \lambda|}=2 \frac{\int_{0}^{1} a(x)\left|f^{\prime}(x)\right|^{2} d x}{\int_{0}^{1} b(x)\left|f^{\prime}(x)\right|^{2} d x} \leq 2 \max _{0 \leq x \leq 1} \frac{a(x)}{b(x)} .
$$

So $\Lambda_{0}$ is a bounded set of $\mathbb{C}$ and there is no eigenvalue on the imaginary axis. These together with (i) of Theorem 3.8 show that $\operatorname{Re} \lambda \leq-\alpha$ for some $\alpha>0$ for all $\lambda \in \sigma(\mathcal{A})$.

It is noticed that we only get the asymptotic expression for larger eigenvalues. From constant case that both $a$ and $b$ are constant, there is a sequence of finite eigenvalues that approach to continuous spectrum. However, for variable $a, b$, it becomes complicated that needs further investigations.

Acknowledgements The authors would like to thank anonymous referees for their careful reading and critical comments that helped us improving the original manuscript substantially. The supports of the Natural Science Foundation of China and the National Research Foundation of South Africa are gratefully acknowledged.

\section{References}

[1] H. T. Banks, K. Ito, and Y. Wang, Well posedness for damped second order systems with unbounded input operators, Differ. Integral Equ. 8, 587-606 (1995).

[2] G. D. Birkhoff, On the asymptotic character of the solutions of certain linear differential equations containing a parameter, Trans. Am. Math. Soc. 9, 219-231 (1908).

[3] W. E. Boyce and R. C. DiPrima, Elementary Differential Equations and Boundary Value Problems, 3rd Edition, (Wiley, New York, 1977).

[4] S. Chen, K. S. Liu, and Z. Liu, Spectrum and stability of elastic systems with global or local Kelvin-Voigt damping, SIAM J. Appl. Math. 59, 651-668 (1998).

[5] H. Cheng, Advanced Analytic Methods in Applied Mathematics, Science, and Engineering (Luban Press, 2007).

[6] N. Dunford and J. T. Schwartz, Linear Operators. Part II, Spectral Theory (Wiley-Interscience, New York, 1967).

[7] W. N. Everitt, M. K. Kwong, and A. Zettl, Oscillation of eigenfunctions of weighted regular Sturm-Liouville problems, J. Lond. Math. Soc. 27(2), 106-120 (1983).

[8] I. Gohberg, S. Goldberg, and M. A. Kaashoek, Classes of Linear Operators, Vol. I (Birkhäuser, Basel, 1990).

[9] B. Z. Guo, Riesz basis approach to the stabilization of a flexible beam with a tip mass, SIAM J. Control Optim. 39, 1736-1747 (2001).

[10] B. Z. Guo, Riesz basis property and exponential stability of controlled Euler-Bernoulli beam equations with variable coefficients, SIAM J. Control Optim. 40, 1905-1923 (2002).

[11] B. Z. Guo and H. Zwart, Riesz Spectral System, Preprint, (University of Twente, The Netherlands, 2001).

[12] B. Jacob and C. Trunk, Location of the spectrum of operator matrices which are associated to second order systems, Operators and Matrices 1, 45-60 (2007).

[13] B. Jacob, C. Trunk, and M. Winklmeier, Analyticity and Riesz basis property of semigroups associated to damped vibrations, J. Evol. Equ. 8, 263-281 (2008).

[14] K. S. Liu, Locally distributed control and damping for concervative systems, SIAM J. Control Optim. 35, 1574-1590 (1997). 
[15] K. S. Liu and Z. Liu, Exponential decay of energy of the Euler-Bernoulli beam with locally distributed Kelvin-Voigt damping, SIAM J. Control Optim. 36, 1086-1098 (1998).

[16] K. S. Liu and Z. Liu, Exponential decay of energy of vibrating strings with local viscoelasticity, Z. Angew. Math. Phys. 53, 265-280 (2002)

[17] K. S. Liu and B. Rao, Exponential stability for wave equations with local Kelvin-Voigt damping, C.R. Acad. Sci. I, Math. 339, 769-774 (2004).

[18] Z.H. Luo, B.Z. Guo, and O. Morgul, Stability and Stabilization of Infinite Dimensional Systems with Applications (SpringerVerlag, London, 1999).

[19] M. A. Naimark, Linear Differential Operators, Vols. I, II (Ungar, New York, 1967, 1968).

[20] M. Renardy, On localized Kelvin-Voigt damping, Z. Angew. Math. Mech. 84, 280-283 (2004).

[21] J. M. Wang, B.Z. Guo, and B. Chentouf, Boundary feedback stabilization of a three-layer sandwich beam: Riesz basis approach, ESAIM, Control, Optimisation Calc. Var. 12, 12-34 (2006).

[22] G. Q. Xu and N. E. Mastorakis, Spectrum of an operator arising elastic system with local K-V damping, Z. Angew. Math. Mech. 88, 483-496 (2008). 